\title{
CAPÍTULO 33
}

\section{CARACTERIZAÇÃO QUIÍMICA ELEMENTAR DE MATRIZES BIOLÓGICAS EMPREGANDO A TÉCNICA DE INAA}

Lucio Leonardo ${ }^{1,2}$; Sandra R. Damatto ${ }^{1}$

${ }^{1}$ Centro de Metrologia das Radiações - IPEN-CNEN/SP

Av. Professor Lineu Prestes, 2242

05508-000 São Paulo, SP

lucioleo@ipen.br

${ }^{2}$ Centro Universitário São Camilo

Av. Nazaré, 1501 - Ipiranga

04263-200 São Paulo - SP

\section{RESUMO}

A INAA foi utilizada no estudo do uso de líquens Canoparmelia texana como bioindicador ambiental de radionuclídeos e metais em região impactada por NORM, na proposta de uso da tartaruga de água doce Trachemis scripta elegans como bioindicadora ambiental em áreas urbanas de São Paulo e com o objetivo de caracterização química elementar dos produtos derivados da Nicotiana tabacum L. Foram determinados nas irradiações realizadas no reator IEA-R1 do IPEN, os elementos As, Ba, Br, Ca, Ce, Co, Cr, Cs, Eu, Fe, Hf, K, La, Lu, Na, Nd, Rb, $\mathrm{Sb}, \mathrm{Sc}, \mathrm{Se}, \mathrm{Sm}, \mathrm{Ta}, \mathrm{Tb}, \mathrm{Th}, \mathrm{U}, \mathrm{Yb}$ e Zn usando os materiais de referência lichen, 
folhas de pêssego, folhas de tomate, peixe e mexilhão. Os resultados indicaram a possibilidade do uso da espécie de liquens como bioindicador de radioatividade e metais, pois as concentrações máximas obtidas de $53 \pm 5 \mathrm{mg} \mathrm{kg}^{-1}, 79 \pm 8 \mathrm{mg} \mathrm{kg}^{-1}$, $135 \mathrm{mg} \mathrm{kg}^{-1}$ para os elementos Hf, Ta e $\Sigma$ ETR, foram maiores que as concentrações $1,51 \pm 0,05 \mathrm{mg} \mathrm{kg}^{-1}, 1,70 \pm 0,22 \mathrm{mg} \mathrm{kg}^{-1}$ e $8,5 \mathrm{mg} \mathrm{kg}^{-1}$ da amostra considerada background, respectivamente, indicando o enriquecimento dos elementos citados. Nas amostras da tartaruga de água doce, os referidos elementos químicos foram determinados no casco, tecido epidérmico córneo, músculo, rim, coração e pulmões e as maiores concentrações foram para os elementos $\mathrm{Ca}, \mathrm{K}$ e Na. Nas amostras de escudo epidérmico e casco foi observada a presença de $\mathrm{Zn}$ que pode ser uma característica da espécie ou uma contribuição do ambiente, sendo necessário mais amostras para a verificação da possibilidade de uso da espécie como bioindicador. Nos produtos derivados da Nicotiana tabacum L. comercializados em algumas regiões do Brasil, como cigarros, charutos, fumo de corda e rapé, os elementos que apresentaram maiores concentrações foram $\mathrm{Ba}, \mathrm{Br}, \mathrm{Ca}, \mathrm{Ce}, \mathrm{K}$, e $\mathrm{Zn}$.

\section{INTRODUÇ̃̃o}

As possibilidades de utilização do reator IEA R-1 na aplicação da análise por ativação com nêutrons térmicos têm fomentado várias pesquisas ambientais. Utilizando padrões específicos irradiados juntamente com a matriz estudada e posteriormente realizando-se a espectroscopia gama a partir das medidas em detectores HPGe do Laboratório de Radiometria Ambiental, a caracterização de vários elementos permitiu a conclusão em diversos estudos ambientais. No período de 2007 a 2010, a hipótese de utilização de líquens da espécie Canoparmelia texana como bioindicadora de radioatividade ambiental e metais motivou uma série de medidas gama passiva, alfa e beta total precedidas de um procedimento radioquímico e da técnica de INAA com a utilização do reator IEA-R1 em amostras de líquens e de solo em duas regiões impactadas por NORM. Leonardo e colegas [1-3] apresentaram resultados dessas análises. No período de 2016 a 2018, o reator IEA-R1 foi utilizado novamente para a caracterização elementar da espécie exótica de tartaruga de água doce Trachemis scripta elegans procedente de parques urbanos, recebida eutanasiada da prefeitura da cidade de São Paulo devido à controle populacional. As investigações da possibilidade de utilização de tal espécie como bioindicador ambiental para metais tem sido estudado [4-6]. Além de estudos ambientais, a aplicação da técnica de INAA permitiu contribuição para estudos na área de saúde com a determinação de concentrações significativas de elementos em produtos derivados da planta Nicotiana tabacum L na análise 
dos principais produtos comercializados no Brasil, como cigarros, charutos, fumo de corda e rapé $[7,8]$.

\section{APLICAÇÕES AMBIENTAIS}

O estudo de bioindicadores de poluição ambiental encontra-se desenvolvido em países da Europa [9] e relativamente novo no Brasil. Nas condições ambientais específicas do país e para aplicações não só para metais, como o caso de poluição radioativa, o campo de pesquisa se mostrou inédito e promissor. As particularidades dos liquens e as possibilidades do estudo em tartarugas possibilitam investigação ambiental em regiões impactadas, seja por NORM ou em ambientes urbanos.

\subsection{O Líquen Canoparmelia texana como Bioindicador Ambiental}

Os líquens são uma associação simbiótica entre uma alga ou uma cianobactéria e um fungo. Como a superfície em contato com o ambiente é isenta de uma camada protetora (cutícula) e de camada cerosa, seu conteúdo hídrico varia segundo a umidade ambiente, havendo assim a possibilidade de absorção de partículas de poluição ambiental que se acumula no espaço intercelular [10]. Em ambientes poluídos, a espécie de líquens Canoparmelia texana prevalece em relação às demais espécies e apresenta grande superfície folhosa, ou seja, maior área de absorção.

Dois locais de estudo em regiões impactadas por NORM foram escolhidos: as imediações de indústrias de chumbo e estanho metálico localizado no município de Pirapora do Bom Jesus - SP e nas proximidades das pilhas de fosfogesso, resíduo da produção de fertilizantes fosfatados, em Cubatão - SP.

Os líquens coletados em vários pontos, incluindo o ponto considerado background, foram submetidos à pré-tratamento com a remoção mecânica de impurezas com auxílio de microscópio binocular. Aproximadamente $200 \mathrm{mg}$ de cada amostra e padrões foram irradiados por 16 horas sob fluxo de $(4,5 \pm 0,5) \cdot 10^{12}$ n. $\mathrm{cm}^{-2} \mathrm{~s}^{-1}$ e contados em duas séries de medidas defasadas em uma semana. Com o auxílio dos materiais de referência IAEA-Lichen-336, BEN-Basalt IWG-GIT, USGS MAG-1 e IAEA-Soil 7, foram analisados os elementos As, $\mathrm{Ba}, \mathrm{Br}, \mathrm{Ca}$, $\mathrm{Ce}, \mathrm{Co}, \mathrm{Cr}, \mathrm{Cs}, \mathrm{Eu}, \mathrm{Fe}, \mathrm{Hf}, \mathrm{K}, \mathrm{La}, \mathrm{Lu}, \mathrm{Na}, \mathrm{Nd}, \mathrm{Rb}, \mathrm{Sb}, \mathrm{Sc}, \mathrm{Se}, \mathrm{Sm}, \mathrm{Ta}, \mathrm{Tb}, \mathrm{Th}$, $\mathrm{U}, \mathrm{Yb}$ e $\mathrm{Zn}$.

Dos elementos analisados, as concentrações mais relevantes em comparação aos resultados das amostras consideradas background foram obtidas na região 
impactada por NORM devido a indústria de produção de chumbo e estanho metálico e são apresentados na Tabela 1 a seguir.

Tabela 1 - Concentração $\left(\mathrm{mg} \mathrm{kg}^{-1}\right)$ de Hf, Ta e do $\Sigma E T R$ em amostras de líquens em Pirapora do Bom Jesus e $\Sigma$ ETR em Cubatão

\begin{tabular}{|c|c|c|c|c|}
\hline & \multicolumn{3}{|c|}{ Pirapora do Bom Jesus } & Cubatão \\
\hline & $\begin{array}{c}\text { Hf } \\
\left(\mathbf{m g ~ k g}^{-1}\right)\end{array}$ & $\begin{array}{c}\text { Ta } \\
\left(\mathbf{m g ~ k g} \mathbf{~ g}^{-1}\right)\end{array}$ & $\begin{array}{c}\mathbf{\Sigma} \text { ETR } \\
\left(\mathbf{m g ~ k g}^{-1}\right)\end{array}$ & $\begin{array}{c}\mathbf{\Sigma} \text { ETR } \\
\left(\mathbf{m g ~ k g}^{-1}\right)\end{array}$ \\
\hline Conc. máxima & $53 \pm 5$ & $79 \pm 8$ & 135 & 146 \\
\hline Concentração $b g$ & $1,51 \pm 0,05$ & $1,70 \pm 0,22$ & 8,5 & 16 \\
\hline
\end{tabular}

\subsection{Estudos da tartaruga de água doce Trachemis scripta elegans como bioindicadora ambiental}

A espécie de tartaruga de água doce Trachemys scripta elegans (Wied-Neuwied, 1839) é exótica, de origem americana, sendo introduzida comercialmente de modo ilegal no Brasil como animal de estimação. Considerada invasora, a agressividade característica de animais adultos faz com que os mesmos sejam costumeiramente abandonados pelos proprietários em parques das cidades, geralmente em locais que podem estar contaminados. De fácil adaptação, essa espécie pode competir com espécies nativas motivando a prefeitura a realizar um controle. $\mathrm{O}$ estudo visou avaliar a concentração de elementos acumulados nos animais para detectar possíveis contaminantes presentes nos corpos d'água que possam vir a interferir na saúde humana e qualidade ambiental. Para tanto, amostras de tecidos de 10 fêmeas doadas pelo DEPAVE, já eutanasiadas e congeladas, foram submetidas na técnica INAA utilizando materiais de referência IAEA-Lichen - 336, Tomato Leaves do NIST e Soil 3 da IAEA.

Os resultados obtidos indicam concentrações características nos diferentes tecidos devido suas funções específicas. A concentração relevante encontrada foi do elemento $\mathrm{Zn}$ (valor máximo $255 \pm 8 \mathrm{mg} \mathrm{kg}^{-1}$ ) em amostras de escudo epidérmico que, em comparação a $64 \pm 2 \mathrm{mg} \mathrm{kg}^{-1}$ de $\mathrm{Zn}$ encontrado no osso e do valor de referência da Crosta Continental Superior $52 \mathrm{mg} \mathrm{kg}^{-1}$. O resultado pode indicar uma característica da espécie ou uma ação antrópica no ambiente, necessitando de mais estudos. 


\subsection{Caracterização Química Elementar dos Produtos Derivados da Nicotiana tabacum L}

Os derivados do Nicotiana tabacum L são os produtos comercializados mais consumidos no mundo, por exemplo, o cigarro, o charuto e o narguilé [11,12]; entre esses produtos destaca-se o cigarro, por ser o mais consumido e ser considerado um dos produtos de consumo mais lucrativos do mundo. Os efeitos danosos que estes produtos causam a saúde humana são há anos intensivamente discutidos e muitos estudos são realizados mundialmente relacionando o uso dos derivados do tabaco com diversas doenças [13-15].

A fumaça do cigarro é constituída de duas fases, a particulada, que é composta por nicotina e alcatrão, sendo esse último composto de várias substâncias cancerígenas, e a fase gasosa, composta por substâncias tóxicas, como monóxido de carbono, dióxido de carbono, óxidos de nitrogênio, amônia, nitrosamidas voláteis, cianeto de hidrogênio, compostos voláteis de enxofre e hidrocarbonetos, As, Ni, Cd, substâncias radioativas como o ${ }^{210} \mathrm{~Pb}$ e ${ }^{210} \mathrm{Po}$, entre outros $[16,12,17]$.

No estudo dos derivados da N. tabacum L. foram analisados os cigarros convencionais, cigarros aromatizados, cigarros de palha, charutos, rapés e fumo de corda desfiado e em rolo (Tabela 2), totalizando 82 amostras. As diversas amostras foram adquiridas em estabelecimentos comerciais públicos e os elementos As, $\mathrm{Ba}, \mathrm{Br}, \mathrm{Ca}, \mathrm{Ce}, \mathrm{Co}, \mathrm{Cr}, \mathrm{Fe}, \mathrm{K}, \mathrm{La}, \mathrm{Na}, \mathrm{Rb}, \mathrm{Sm}, \mathrm{Sc}$, Th e Zn, foram determinados pela técnica de INAA. Os maiores valores de concentração foram obtidos para os elementos $\mathrm{Ca}, \mathrm{K}, \mathrm{Ba}, \mathrm{Br}$, Ce e $\mathrm{Zn}$ e as amostras que apresentaram as maiores concentrações desses elementos foram os de cigarros de palha e fumos de corda $[7,8]$.

Tabela 2 - Concentração obtidas nos derivados do Nicotiana tabacum L

\begin{tabular}{|c|c|c|c|c|c|}
\hline Cig. não aromatizado & Cig. aromatizado & Cig. de palha & Charuto & Fumo de corda \\
\hline Ca (\%) & 3,1 & 2,4 & 3,6 & 4,1 & 2,5 \\
\hline K (\%) & 3,5 & $<0,3$ & 2,4 & 3,8 & 3,5 \\
\hline Ba (mg kg-1) & 140 & 121 & 112 & 102 & 123 \\
\hline Br (mg kg-1) & 56 & 49 & 55 & 111 & 67 \\
\hline Zn (mg kg-1) & 38 & 38 & 64 & 68 & 74 \\
\hline Ce (mg kg-1) & 2,9 & 1,8 & 6,1 & 1,8 & 5,4 \\
\hline
\end{tabular}

\section{CONCLUSÕES}

Matrizes biológicas podem fornecer informações sobre possíveis contaminações ambientais por elementos químicos inorgânicos e consequentemente dados relevantes para a saúde humana. A técnica analítica de análise por ativação com 
nêutrons instrumental tem se mostrado eficaz nas determinações dos principais elementos de interesse em estudos ambientais.

\section{REFERÊNCIAS}

1. LEONARDO, L.; MAZZILLI, B. P.; DAMATTO, S. R.; SAIKI, M.; OLIVEIRA, S. M. B. Assessment of atmospheric pollution in the vicinity of a tin and lead industry using lichen species Canoparmelia texana. Journal of Environmental Radioactivity, v. 102, p. 906-910, 2011.

2. LEONARDO, L.; DAMATTO, S. R.; GIOS, B. R.; MAZZILLI, B. P. Lichen specie Canoparmelia texana as bioindicator of environmental impact from the phosphate fertilizer industry of São Paulo, Brazil. Journal of Radioanalytical and Nuclear Chemistry, v. 299, p.1935-1941, 2013.

3. MAZZILLI, B. P.; LEONARDO, L.; DAMATTO, S. R. Monitoring anthropogenic airborne natural radionuclides in the vicinity of a TENORM industry using lichen as bio-indicator. $I n$ : $6^{\text {th }}$ INTERNATIONAL SYMPOSIUM ON NATURALLY OCCURRING RADIOACTIVE MATERIAL (NORM VI), 2010, Marrakech. Anais [...], 2010. v. 1, p. 66-72.

4. SARTORI, L. P.; LEONARDO, L.; DAMATTO, S. R.; SANTOS, N. D.; AUGUSTO, V. M. Use of exotic turtle (Trachemys scripta elegans) as bioindicators of trace elements from the urban area of São Paulo. In: $2^{\text {nd }}$ PAN-AMERICAN CONFERENCE FOR ALTERNATIVE METHODS, 2018, Rio de Janeiro-RJ. Anais [...],2018.

5. SANTOS, N. D. D.; AUGUSTO, V. M.; SARTORI, L. P.; LEONARDO, L.; DAMATTO, S. R. Caracterização elementar em amostras de tartarugas de água doce Trachemis scripta elegans. In: III CONGRESSO MULTIPROFISSIONAL, 9-10 nov. 2018, São Paulo-SP. Anais [...]. São Paulo: Centro Universitário São Camilo. Disponível em: https://saocamilo-sp.br/assets/uploads/AF_ Anais_ $3^{\circ}$ Congresso.pdf.

6. LEONARDO, L. et al. Metais determinados na espécie de cágado Trachemys scripta elegans Wied, 1839 abandonadas em área urbana de São Paulo-SP. In: $18^{\circ}$ ENCONTRO NACIONAL DE QUÍMICA ANALÍTICA, 18-21 set. 2016, Florianópolis-SC. Anais [...], 2016.

7. OLIVEIRA, A. S. G. R.; DAMATTO, S. R. Estudo da composição elementar de derivados de tabaco pela Análise por Ativação com Nêutrons Instrumental. In: XVI CONGRESSO BRASILEIRO DE GEOQUÍMICA, 22-25 ago. 2017, Búzios-RJ. Anais [...], 2017. 
8. OLIVEIRA, A. S. G. R. Radioatividade natural, elementos maiores e traços determinados em produtos nacionais derivados da Nicotiana tabacum L. 2017. 174 p. Dissertação (Mestrado em Tecnologia Nuclear - Aplicações) - Instituto de Pesquisas Energéticas e Nucleares (IPEN), Universidade de São Paulo (USP), São Paulo, 2017. DOI: 10.11606/D.85.2017.tde-13122017-105430.

9. CONTI, M. E.; CECCHETTI, G. Biological monitoring: lichens as bioindicators of air pollution assessment: a review. Environmental Pollution, v. 114, p. 471-492, 2001.

10. GARTY, J.; GALUN, M.; KESSEL, M. Localization of heavy metals and other elements accumulated in the lichen thallus. The New Phytologist, v. 82, p. 159-168, 1979.

11. KHATER, A. E. M.; ABD EL-AZIZ, N. S.; AL-SEWAIDAN, H. A.; CHAOUACHI, K. Radiological hazards of Narghile (hookah, shisha, goza) smoking: activity concentrations and dose assessment. J. Environ. Radioac. v. 99, p. 1808-1814, 2008.

12. PRATA, V. M.; EMÍDIO, E. S.; DÓREA, H. D. Aplicação de métodos quimiométricos na caracterização de charutos utilizando extração por dispersão da matriz em fase sólida e análise por cromatografia gasosa acoplada à espectrometria de massas. Quim. Nova, v. 34, n. 1, p. 53-58, 2011.

13. ARNSON, Y.; SHOENFELD, Y.; AMITAL, H. Effects of tobacco smoke on immunity, inflammation and autoimmunity. J. Autoim, v. 34, p. J258-J265, 2010.

14. MORTENSEN, M. E.; WONG, L-Y.; OSTERLOH, J. D. Smoking status and urine cadmium above levels associated with subclinical renal effects in U.S. adults without chronic kidney disease. Int. J. Hyg. Environ. Heal., v. 214, p. 305310, 2011.

15. DELA CRUZ, C. S.; TANOUE, L. T.; MATTHAY, R. A. Lung cancer: epidemiology, etiology, and prevention. Clin. Chest. Med., v. 32, p. 605-644, 2012.

16. VERMA, S.; YADAV, S.; SINGH, I. Trace metal concentration in different Indian tobacco products and related health implications. Food Chem. Toxic., v. 48, p. 2291-2297, 2010.

17. DUAN, J.; HUANG, Y.; LI, Z.; ZHENG, B.; LI, Q.; XIONG, Y.; WU, L. MIN, S. Determination of 27 chemical constituents in Chinese southwest tobacco by FT-NIR spectroscopy. Ind. Crops Prodc., v. 40, p. 21-26, 2012.

18. WEDEPOHL, K. H. The composition of the continental crust. Geochim. Cosmochim. Acta, v. 59, n. 7, p. 1217-1232, 1995. 
